\title{
Degree of Freedom Multi-axle Vehicle Steering Characteristic Analysis Yang $\mathrm{M}$.
}

\author{
College of Mechanical Engineering and Automation, University of Science and Technology \\ Liaoning, Anshan, China
}

annalily@126.com

Keywords: component; multi-axle vehicle; steeering;control; analysis

\begin{abstract}
Steering system stability of multi-axle vehicle mainly depends on the system's center of gravity position and the tire cornering stiffness parameters of the bridge. Zero side-slip Angle proportional control strategy is used, after many bridge steering system under arbitrary speed can guarantee system steady-state value is zero side-slip angle, lateral acceleration steady-state value are reduced greatly, and make the yawing angular velocity in high speed changed little compared with low speed, the driver does not need to change driving habits. Frequency response of the system, on the other hand, has greatly improved and react more quickly, to process more smoothly, significantly improve the direction of the driving stability and lateral stability.
\end{abstract}

\section{Introduction}

With the need of construction and production development, heavy multi-axle vehicles have found an increasingly wide application, and multi-axle steering performance directly influences the vehicle's maneuvering stability, active safety and economic efficiency, how to improve the steering performance is one of the significant technologies for heavy multi-axle vehicles. So far, there are quite a few open foreign literatures about multi-axle steering technology, while domestic literatures mainly focus on the optimization of vehicle steering linkage's geometric dimensions and locations, with a few on the research of multi-axle steering strategy, those literatures usually tend to analyze multi-axle vehicle's steering characteristics and propose multi-axle steering control strategy on the basis of simplified linear model, meanwhile neglect the drive's subjective feedback role. Considering the above factors, this paper makes a further more research on the multi-axle steering control strategy.

On the basis of existing theories, this paper derives linear 3DOF dynamic model for multi-axle vehicle according to Lagrange Equation, through proper transformation,

\section{Model}

Multi-axle vehicle 3 degree of freedom dynamic model

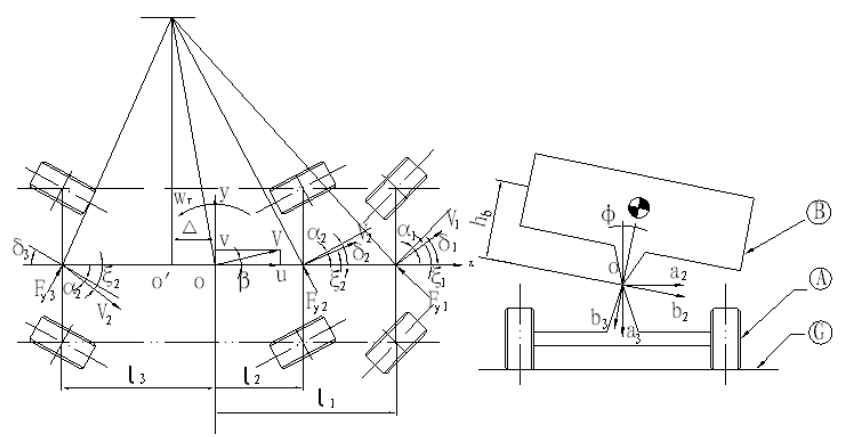

Fig.1 3 degree of freedom dynamic model

Multi-axle vehicle 3 degree of freedom dynamic model includes longitudinal movement along Y axis, yaw movement across $\mathrm{Z}$ axis, and roll movement across $\mathrm{X}$ axis. According to Lagrange equation, the simplified model of the multi-axle vehicle is as follows 


$$
\left\{\begin{array}{l}
\frac{d}{d t}\left(\frac{\partial E_{T}}{\partial v}\right)+w_{r} \frac{\partial E_{T}}{\partial u}=\sum F_{Y i} \\
\frac{d}{d t}\left(\frac{\partial E_{T}}{\partial \gamma}\right)+u \frac{\partial E_{T}}{\partial v}-v \frac{\partial E_{T}}{\partial u}=M_{Z} \\
\frac{d}{d t}\left(\frac{\partial E_{T}}{\partial \dot{\phi}}\right)-\frac{\partial E_{T}}{\partial \phi}+\frac{\partial E_{V}}{\partial \phi}+\frac{\partial E_{D}}{\partial \dot{\phi}}=\sum d_{i} F_{Y i}
\end{array}\right.
$$

in which, $E_{T}$ is the total kinetic energy; $E_{V}$ is the total potential energy; $E_{D}$ is the total dissipated energy; $w_{r}$ is the yaw rate, $\mathrm{rad} / \mathrm{s} ;{ }^{v}$ is the longitudinal velocity,m/s; $u$ is the side velocity, $\mathrm{m} / \mathrm{s} ;{ }_{Y i}$ is the side force of i axle, $\mathrm{N} ; M_{Z}$ is the total yaw torque, N.m; ${ }^{\phi}$ is the roll angle of the vehicle, rad; $d_{i}$ is the lateral offset of $\mathrm{i}$ axle caused by roll angle of the vehicle, $\mathrm{m}$;

Differential equations of the multi-axle vehicle as

$$
\left\{\begin{array}{l}
M\left(\dot{v}+u w_{r}\right)+\left(\sum_{i=1}^{n} m_{i} l_{i}\right) \dot{w}_{r}-m_{b} h_{b} \dot{p}= \\
\sum_{Y i} F_{i=1}=\sum_{i} k_{i}\left(\beta+\frac{l_{i}}{u} w_{r}-\delta_{i}\right) \\
\left(\sum_{i=1}^{n} m_{i} l_{i}\right)\left(\dot{v}+u w_{r}\right)+I_{Z Z} \dot{w}_{r}+I_{X Z b} \dot{p}= \\
\sum_{Y i} F_{Y i} l_{i}+M_{d}=\sum_{i=1}^{n} k_{i}\left(\beta+\frac{l_{i}}{u} w_{r}-\delta_{i}\right) l_{i}+M_{d} \\
I_{X X} \dot{p}+C_{\varphi} p+\left(K_{\varphi}-m_{b} g h_{b}\right) \varphi-m_{b} h_{b}\left(\dot{v}+u w_{r}\right)+I_{X Z b} \dot{w}_{r} \\
=\sum d_{i} F_{Y i}=\sum_{i=1}^{n} d_{i} k_{i}\left(\beta+\frac{l_{i}}{u} w_{r}-\delta_{i}\right)
\end{array}\right.
$$

in which,

$I_{Z Z}=\sum_{i=1}^{n}\left(m_{i} l_{i}^{2}+I_{Z Z i}\right)+I_{Z Z b}$ is the yaw moment of inertia, kg. $\mathrm{m}^{2}$

$M=\sum_{i=1}^{n} m_{i}+m_{b}, \mathrm{~m} ;$

$I_{X X}=I_{X X b}+m_{b} h_{b}^{2}$ is the roll moment of inertia, kg.m ${ }^{2}$;

$k_{i}$ isComprehensive cornering stiffness of I axel, N/rad;

$\beta$ is sideslip angle, rad;

$\delta_{i}$ is the turning angle of wheel.

The relationship of the wheel turning angle between the first axle and the Nth axle is

$$
\delta_{i}=\frac{\left(l_{i}-l_{1}\right) \delta_{n}+\left(l_{n}-l_{i}\right) \delta_{1}}{l_{n}-l_{1}}
$$

Equation of state is 


$$
\begin{aligned}
& {\left[\begin{array}{cccc}
\sum_{i=1}^{n} m_{i} l_{i} & u M & -m_{b} h_{b} & 0 \\
I_{Z Z} & u \sum_{i=1}^{n} m_{i} l_{i} & I_{X Z b} & 0 \\
I_{X Z b} & -u m_{b} h_{b} & I_{X X} & 0 \\
0 & 0 & 0 & 1
\end{array}\right]\left[\begin{array}{c}
\dot{w}_{r} \\
\dot{\beta} \\
\dot{p} \\
\dot{\varphi}
\end{array}\right]+} \\
& {\left[\begin{array}{cccc}
u M-\frac{\sum_{i=1}^{n} k_{i} l_{i}}{u} & -\sum_{i=1}^{n} k_{i} & 0 & 0 \\
u \sum_{i=1}^{n} m_{i} l_{i}-\frac{\sum_{i=1}^{n} k_{i} l_{i}^{2}}{u} & -\sum_{i=1}^{n} k_{i} l_{i} & 0 & 0 \\
-u m_{b} h_{b}-\frac{\sum_{i=1}^{n} k_{i} l_{i} d_{i}}{u} & -\sum_{i=1}^{n} k_{i} d_{i} & C_{\varphi} & K_{\varphi}-m_{b} g h_{b} \\
0 & 1 & 0
\end{array}\right]\left[\begin{array}{c}
w_{r} \\
\beta \\
p \\
\varphi
\end{array}\right]=\left[\begin{array}{c}
-\sum_{i=1}^{n} k_{i} \delta_{i} \\
-\sum_{i=1}^{n} k_{i} l_{i} \delta_{i} \\
-\sum_{i=1}^{n} k_{i} d_{i} \delta_{i} \\
0
\end{array}\right]+\left[\begin{array}{l}
0 \\
1 \\
0 \\
0
\end{array}\right] M_{d}}
\end{aligned}
$$

in which, $X=\left[w_{r}, \beta, p, \phi\right]^{T}$.

Then, the state equation and output equation are

$$
\dot{X}_{1}=A X_{1}+B U
$$

$$
Y_{1}=C X_{1}
$$

In which, $X_{1}=\left[w_{r}, \beta, p, \phi\right]^{T}, U=\left[\delta_{1}, \delta_{n}, M_{d}\right]^{T}, Y_{1}=\left[w_{r}, \beta, p, \phi\right]^{T}$.

$$
A=-P \times\left[\begin{array}{cccc}
u M-\frac{\sum_{i=1}^{n} k_{i} l_{i}}{u} & -\sum_{i=1}^{n} k_{i} & 0 & 0 \\
u \sum_{i=1}^{n} m_{i} l_{i}-\frac{\sum_{i=1}^{n} k_{i} l_{i}^{2}}{u} & -\sum_{i=1}^{n} k_{i} l_{i} & 0 & 0 \\
-u m_{b} h_{b}-\frac{\sum_{i=1}^{n} k_{i} l_{i} d_{i}}{u} & -\sum_{i=1}^{n} k_{i} d_{i} & C_{\phi} & K_{\phi}-m_{b} g h_{b} \\
0 & 0 & 1 & 0
\end{array}\right]
$$




$$
\begin{aligned}
C & =\left[\begin{array}{llll}
1 & 0 & 0 & 0 \\
0 & 1 & 0 & 0 \\
0 & 0 & 1 & 0 \\
0 & 0 & 0 & 1
\end{array}\right] \\
P & =-\left[\begin{array}{cccc}
\sum_{i=1}^{n} m_{i} l_{i} & u M & -m_{b} h_{b} & 0 \\
I_{Z Z} & u \sum_{i=1}^{n} m_{i} l_{i} & I_{X Z b} & 0 \\
I_{X Z b} & -u m_{b} h_{b} & I_{X X} & 0 \\
0 & 0 & 0 & 1
\end{array}\right]^{-1}
\end{aligned}
$$

\section{Analysis}

\section{A. Simulation}

Simulink simulation model is set up using MATLAB software based on the equations of multiaxle vehicle dynamic system. The characteristic of the vehicle are different under different control rule.

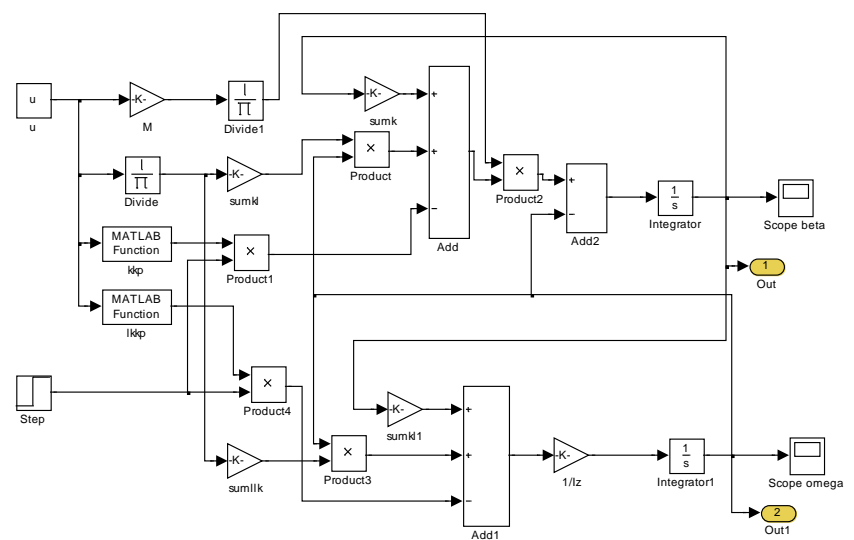

Fig. 2 simulation model

\section{B. Analysis}

Fig. 3 shows that the time domain features of the multi-axle vehicle. (a)The steady-state value equals 0 . The purpose of controlling is achieved. The stability of the vehicle has been improved under MAS mode. (b)The yaw rate is not sensitive to velocity, steering process is more stable. (c) Reversing phase at low speed, lateral acceleration of MAS mode approximately equals to FWS mode. Lateral stability is improved.

Fig. 4 shows that the frequency domain features of the multi-axle vehicle. Magnitude frequency characteristic and phase frequency characteristic are contained. (a)Phase difference of MAS mode reduces half, and the system is more responsive. (b)Phase lag of the yaw rate is not influenced in MAS mode. (c)The response in MAS mode is better than FWS, especially in the middle of the process. 


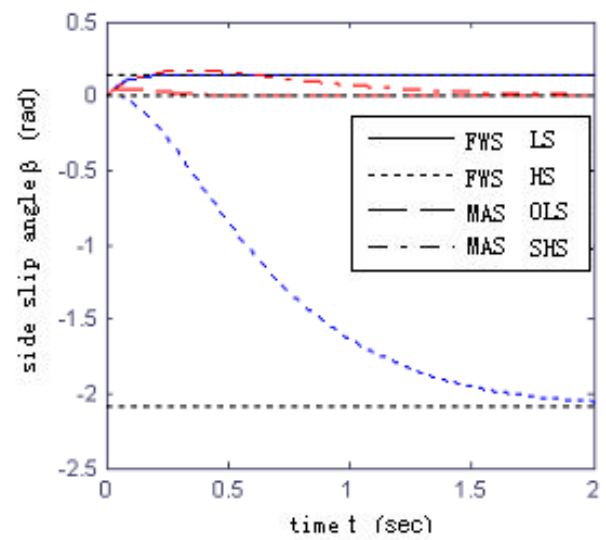

(a)

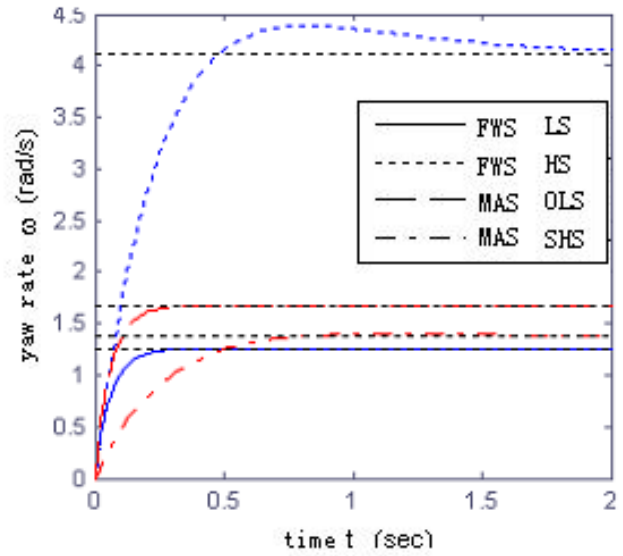

(b)

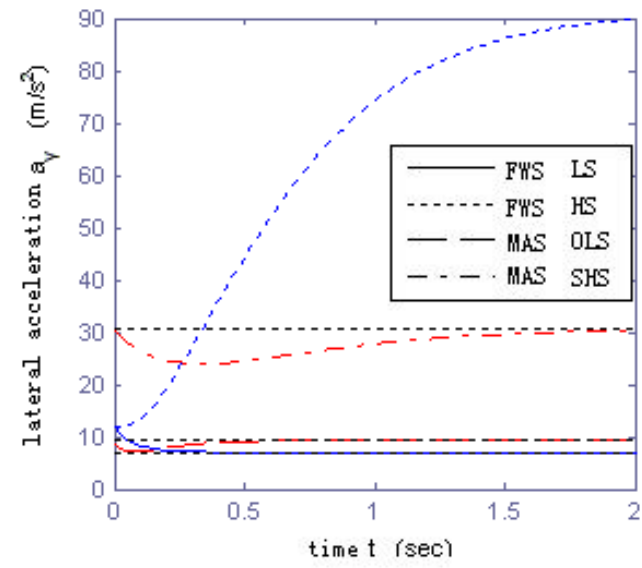

(c)

Fig.3 time domain features 


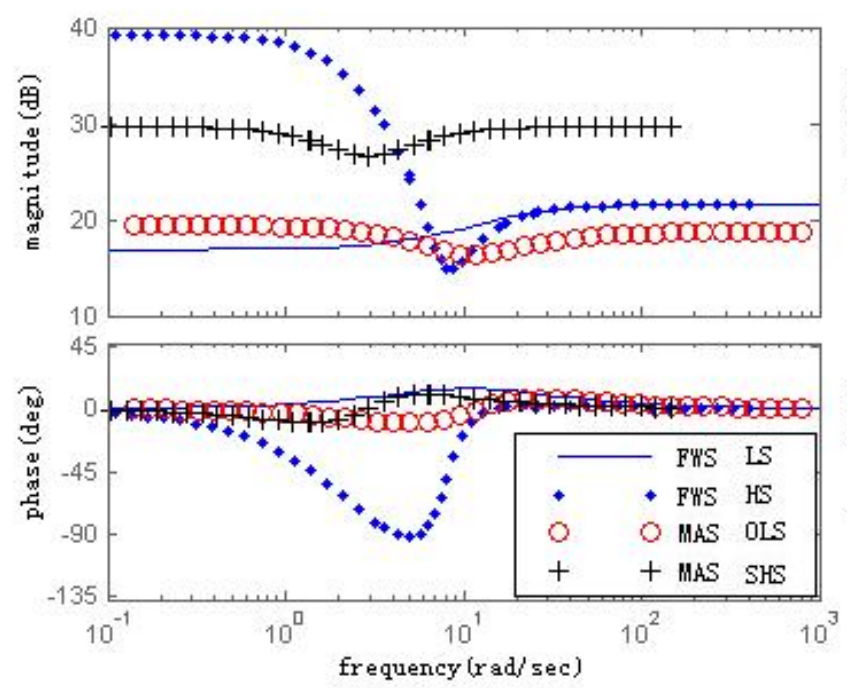

(a)

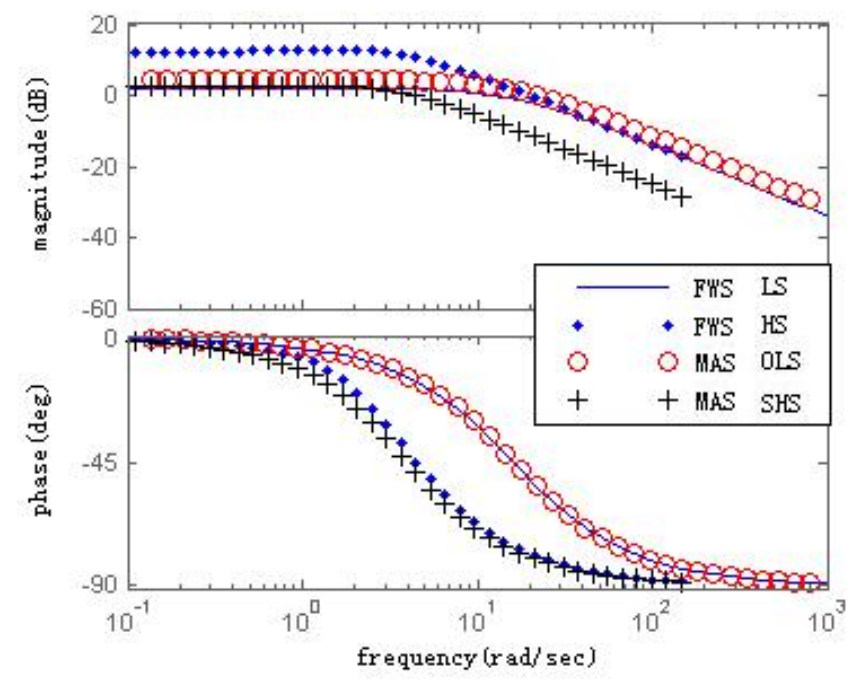

(b)

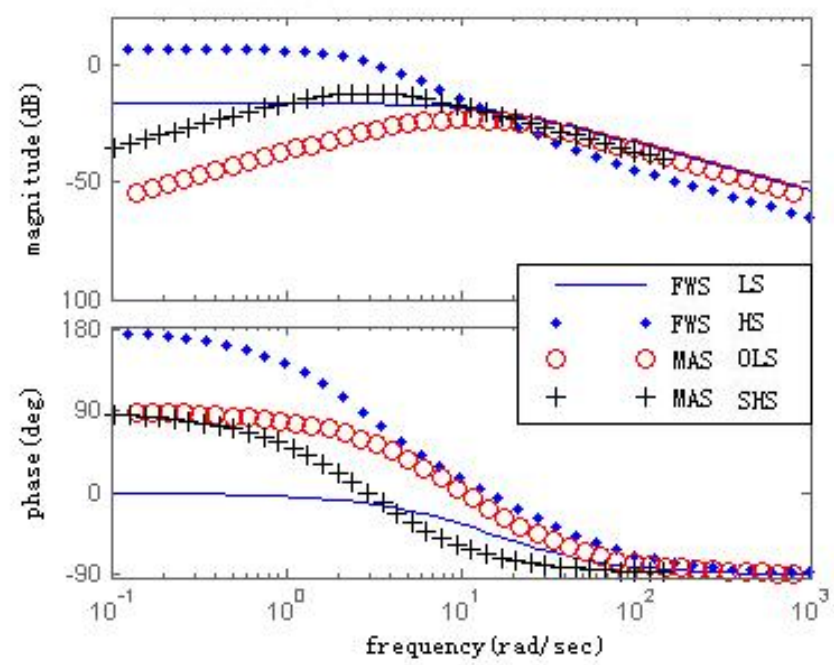

(c)

Fig.4 frequency domain features

\section{Conclusion}

Steering system stability of multi-axle vehicle mainly depends on the system's center of gravity position and the tire cornering stiffness parameters of the bridge. Zero side-slip Angle proportional 
control strategy is used, after many bridge steering system under arbitrary speed can guarantee system steady-state value is zero side-slip angle, lateral acceleration steady-state value are reduced greatly, and make the yawing angular velocity in high speed changed little compared with low speed, the driver does not need to change driving habits. Frequency response of the system, on the other hand, has greatly improved and react more quickly, to process more smoothly, significantly improve the direction of the driving stability and lateral stability.

\section{Acknowledgment}

Thanks to Liaoning Province Department of Education Fund.

\section{References}

[1] K Watanabe, J Yamakawa, $M$ Tanaka,etc.Turing Characteristics of Multi-axle Vehicles[J].Journal of Terramechanics,2007,(44):81-87.

[2] K Bayer, Y S Unlusoy. Steering Strategies for Multi-axle Vehicles [J]. Int J Heavy Vehicle Systems,2008,15(2/3/4):208-236.

[3] G D Yin, $N$ Chen, J X Wang, et al. Robust Control for 4WS vehicles Considering a Varying Tire-road Friction Coefficient [J]. International Journal of Automotive Technology,2010,11(1):33-40.

[4] G D Yin, N Chen, P Li. Improving Handling Stability Performance of Four-wheel Steering Vehicle via $\mu$-synthesis Robust Control[J].IEEE Transactions On Vehicular Technology, 2007, 56(5):2432-2339.

[5] H M LV, N Chen, P Li. Multi-objective $H$ o Optimal Control for Four-wheel Steering Vehicle Based on Yaw Rate Tracking[J].Proc.Instn Mech.Engrs, Part D:J. Automobile Engineering,2004,218(10):1117-1124. 